\title{
Direct endoscopic necrosectomy in infected pancreatic necrosis using lumen-apposing metal stents: Early intervention does not compromise outcome
}

\section{(ㄷ)(우우}

Authors

David Albers ${ }^{1}$, Alexander Meining ${ }^{2}$, Alexander Hann², Younan Kabara Ayoub ${ }^{3}$, Brigitte Schumacher ${ }^{1}$

Institutions

1 Department of Gastroenterology, ElisabethKrankenhaus Essen, academic hospital of the University of Duisburg-Essen, Germany

2 Department of Gastroenterology, University Hospital Wuerzburg, Germany

3 Department of Gastroenterology, University Hospital Cairo, Egypt

submitted 17.8.2020

accepted after revision 23.11 .2020

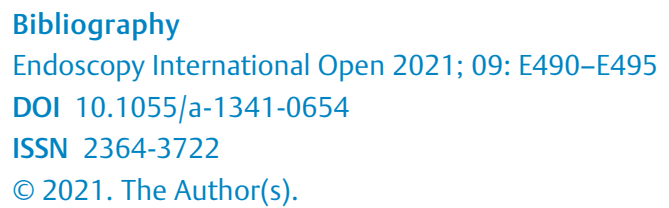
Commons Attribution-NonDerivative-NonCommercial License, permitting copying and reproduction so long as the original work is given appropriate credit. Contents may not be used for commecial purposes, or adapted, remixed, transformed or built upon. (https://creativecommons.org/licenses/by-nc-nd/4.0/)

Georg Thieme Verlag KG, Rüdigerstraße 14,

70469 Stuttgart, Germany

Corresponding author

David Albers, MD, Department of Gastroenterology,

Elisabeth-Krankenhaus Essen, Klara-Kopp-Weg 1, 45138

Essen, Germany

Fax: $+49-2018973119$

d.albers@contilia.de

\section{ABSTRACT}

Background and study aims Infection of pancreatic necrosis is a dreaded complication requiring an intervention. Nevertheless, the optimal timing of the first intervention is unclear, and consensus data are sparse. This retrospective two-center study evaluated direct endoscopic necrosectomy using lumen apposing metal stents in case of proven or suspected infected pancreatic necrosis in an early stage of the disease.

Patients and methods Forty-nine patients with infected pancreatic necrosis were included. Sequent direct endoscopic necrosectomies after lumen apposing metal stent insertion (LAMS) were performed until the resolution of necrosis. In all patients, the first endoscopic intervention was performed within the first 30 days after first proof of pancreatic necrosis. Primary outcome parameters were inflammatory activity, days spent in the Intensive Care Unit (ICU), and mortality.

Results The patient cohort received median 4 necrosectomies (3-5) after a median of 7 days (3-11) after first proof of pancreatic necrosis. Technical and clinical success were achieved in $98.3 \%$ and $87.8 \%$, respectively; the mortality rate was $8.2 \%$. The median C-reactive protein level decreased from $241 \mathrm{mg} / \mathrm{L}$ (182.9-288.9) before the intervention to a median of $23.3 \mathrm{mg} / \mathrm{L}(18-60)$ after therapy. The median time period in the ICU was 5 days (3-9).

Conclusions Early endoscopic therapy in the form of direct endoscopic necrosectomy after LAMS placement within the first 30 days after proof of pancreatic necrosis is effective and does not result in poor outcome. Our retrospective data suggest that early intervention before walled-off necrosis is formed is tenable when it is essential due to the patient's clinical deterioration.

\section{Introduction}

In acute pancreatitis, $15 \%$ to $20 \%$ of patients develop necrosis of pancreatic parenchyma and/or peripancreatic tissue, indicating a severe course of the disease [1-3]. Approximately onethird of these patients develop infected pancreatic necrosis
(IPN), a life-threatening local condition that is a prerequisite for subsequent systemic complication, namely organ failure [4]. Both local and systemic complications account for high morbidity and mortality that usually is fatal without therapeutic intervention. 
Therapeutic procedures, notably open surgery, are accompanied by high mortality rates, particularly when carried out in the first 14 days after onset of the disease [5] Postponing the intervention 4 weeks, after the necrotic collection is demarcated and encapsulated, decreases mortality rate [6, 7].

In parallel to a delayed practice, a paradigm shift in the therapy of pancreatic necrosis, away from open surgery to an interventional step-up approach, has taken place in the last two decades [8].

But in one-quarter of patients, infected pancreatic necrosis can occur during the first 14 days after onset of the disease [9], and early intervention is potentially inevitable.

Current guidelines consider the early infection of pancreatic necrosis and recommend deferring a therapeutic intervention for at least 4 weeks after onset of pancreatitis but advise therapy in case of progressive deterioration of the patient [10].

However, most of the current data are based on surgery and consensus data for optimal timing of the endoscopically based approach are sparse [11]. This retrospective two-center study evaluated early direct endoscopic necrosectomy using lumen apposing metal stents (LAMS) in case of proven or suspected super-infection in comparison to a delayed practice.

\section{Patients and methods}

Between June 2012 and February 2018, all patients endoscopically treated with direct endoscopic necrosectomy after LAMS placement in two German tertiary referral centers were screened with regard to the interval between the first proof of necrosis and the first endoscopic intervention. All patients with an endoscopic treatment within the first 30 days after first proof of necrosis were included in the study. The patient cohort consisted of 49 patients (37 male, 12 female) with a median age of 52.2 years. The etiology of pancreatitis was alcoholic ( $n$ $=17)$, biliary $(n=15)$, hypertriglyceridemia $(n=1)$, post-ERCPpancreatitis $(n=3)$, after surgery $(n=1)$ and unknown $(n=12)$ ( $\triangleright$ Table 1 ).

All patients gave written consent, all procedures performed in the study were ethically in accordance with the 1964 Helsinki Declaration and its later amendments.

Pancreatic necrosis was diagnosed by contrast-enhanced computed tomography (CT) and evaluated with endoscopic ultrasound (EUS). Infected necrosis was confirmed by the presence of gas in the CT scan or was suspected based on clinical and biochemical markers of persistent infection or sepsis, despite maximal therapy in the intensive care unit and without evidence for another infection source.

Technical success was defined as the ability to position and deploy the stent. Clinical success was defined as the resolution of the necrotic collection associated with the recovery of the patient. The periods in the Intensive Care Unit (ICU) and Intermediate Care Unit (IMU) were recorded and C-reactive-protein (CRP) was measured as a marker of inflammatory activity. Concerning adverse events (AEs), bleeding with the need for intervention, perforation, and stent dislocation were recorded. Transfer to surgery was judged as a failure of the procedure.
- Table 1 Patient characteristics.

Patients (n)

Age [median $(95 \% \mathrm{Cl})]$

$52.2(48-59)$

male

$37(75.5 \%)$

Female

$12(24.5 \%)$

Etiology

Alcoholic

$17(34.7 \%)$

Biliary

$15(30.1)$

Post-ERCP

$3(6,1 \%)$

Hypertriglyceridemia

$1(2,0 \%)$

After surgery

$1(2,0 \%)$

Uunknown

$12(24.5 \%)$

Maximum diameter of necrosis $(\mathrm{mm})(95 \% \mathrm{Cl})$

$80(70-90)$

ERCP, endoscopic retrograde cholangiopancreatography.

\section{Endoscopic procedure}

Indication for endoscopic therapy was infected pancreatic necrosis with clinical signs of sepsis. In the case of infected necrosis, a lumen apposing metal stent (Hot-Axios Stent, Boston Scientific, Marlborough, Massachusetts, United States, or NAGI Stent, Taewoong Medical, Wolgot-Myeon, South Korea) was implanted via the transgastral or transduodenal access.

The two stent designs used are $15 \mathrm{~mm}$ in diameter and 10 $\mathrm{mm}$ in length (Boston Scientific) and $16 \mathrm{~mm}$ in diameter and $20 \mathrm{~mm}$ in length (Taewoong medical). In the case of the Hot Axios stent, the drainage of the necrotic cavity was carried out by direct puncture using the electrocautery delivering. In the case of the other stent design, the puncture was performed with a 19G EUS needle (Echotip, Cook Medical, Bloomington, Indiana, United States or Expect, Boston Scientific, Marlborough, Massachusetts, United States). Then a 0.035-inch guidewire (Jagwire Boston Scientific, Marlborough, Massachusetts, United States) was pushed forward, building two loops in the necrotic area. Subsequently, the access to the necrotic cavity was achieved using a cystotome (Ring-Knife, MTW, Wesel, Germany or Boston Scientific, Marlborough, Massachusetts, United States). Thereafter, the access was dilated (CRE Balloon Dilation Catheter, Boston Scientific, Marlborough, Massachusetts, United States), and the stent was deployed. The fluid content of the necrosis was preserved for microbiological testing.

All interventions were performed under monitored anesthesia using propofol or general anesthesia. Concomitant antibiotics (piperacillin/tazobactam or imipenem/cilastin) were administered. In the case of cardiopulmonary instability due to sepsis, the first necrosectomy procedure was postponed for two or three days after stent insertion and was carried out after the recovery of the patient. Otherwise, the first direct endoscopic necrosectomy was performed during the same session immediately after stent placement ( $>$ Fig. 1, $\triangleright$ Fig.2). Sequential endoscopic necrosectomies were performed at in- 


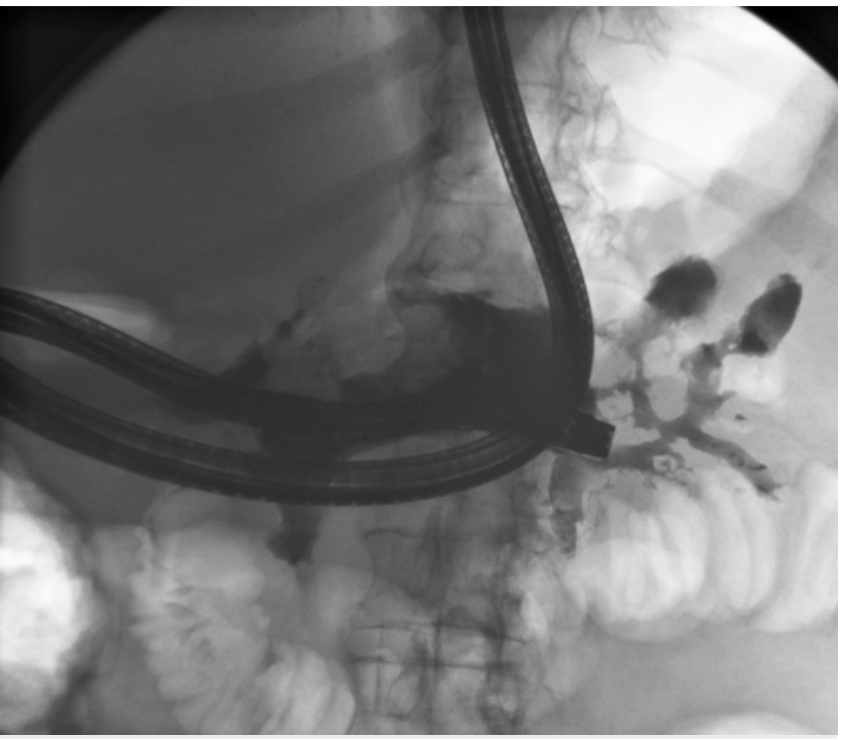

- Fig. 1 Performing direct endoscopic necrosectomy via transduodenal access, contrast agent is applied to visualize the necrotic cavity.

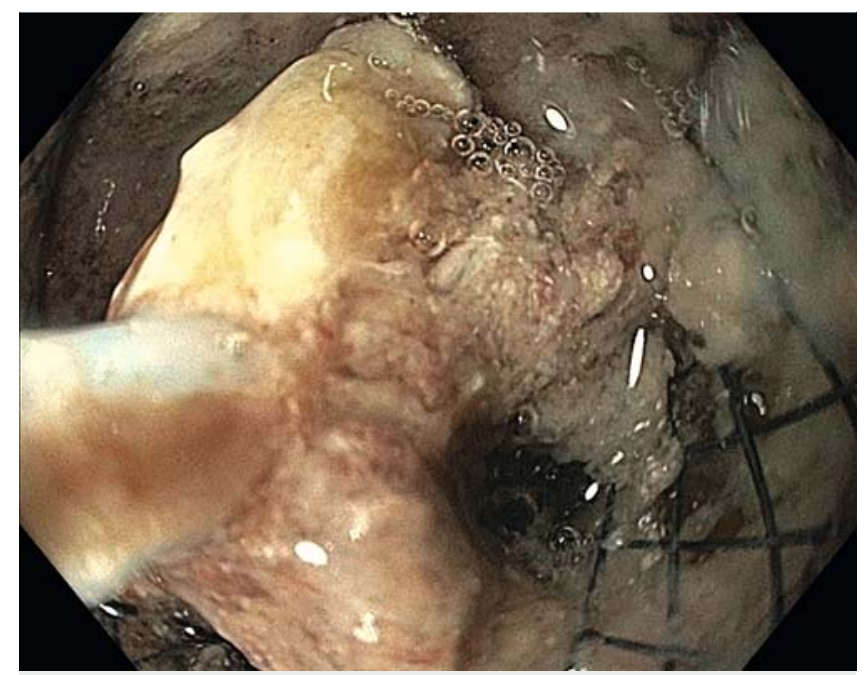

- Fig. 2 First necrosectomy after LAMS placement with the help of an endoscopic snare.

tervals of 2 to 3 days until the complete resolution of the necrotic debris ( $\mathbf{F i g . 3 , ~}$ Fig.4). In five cases (8\%), a second lumen-apposing metal stent was added because of the extent of the necrotic collection. In seven patients (12\%) additional external drainage after the initial endoscopic access was required due to the extension of the necrosis to the lesser pelvis ( Fig. 5, > Fig.6).

\section{Statistical analysis}

Statistics were performed with SPSS version 24 (IBM, United States). The $95 \%$ confidence interval $(\mathrm{Cl})$ of the median was calculated using Bootstrapping. The Wilcoxon signed-rank test was used for continuous variables to investigate differences between paired values. The Mann Whitney $U$ test was used for in-

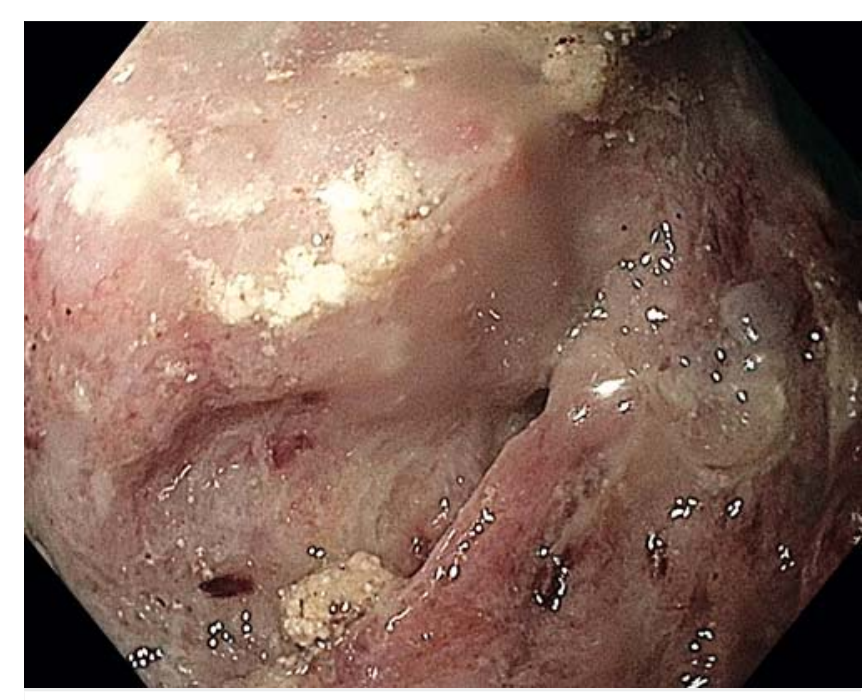

Fig. 3 Necrotic cavity after first necrosectomy.

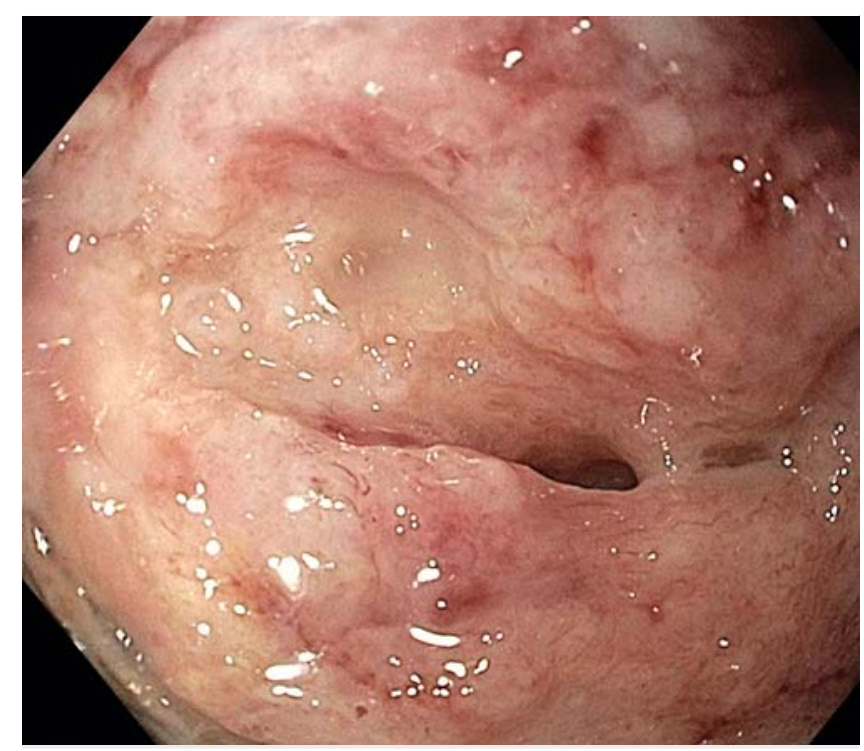

- Fig. 4 Necrotic cavity after last endoscopic necrosectomy.

dependent values. For binominal variables, chi-square was chosen to examine differences between groups. $P<0.05$ indicated statistical significance.

\section{Results}

All 49 patients received a median of four necrosectomies (3-5) after a median of 7 days (3-11) after the first proof of necrosis ( Table 2).

Technical success was defined as the ability to deploy the stent in the correct position; this was achieved in all 49 patients (100\%). The clinical success rate of the procedure defined as clinical resolution of the necrotic collection was $87.8 \%$; the mortality-rate accounted for $8.2 \%$. 


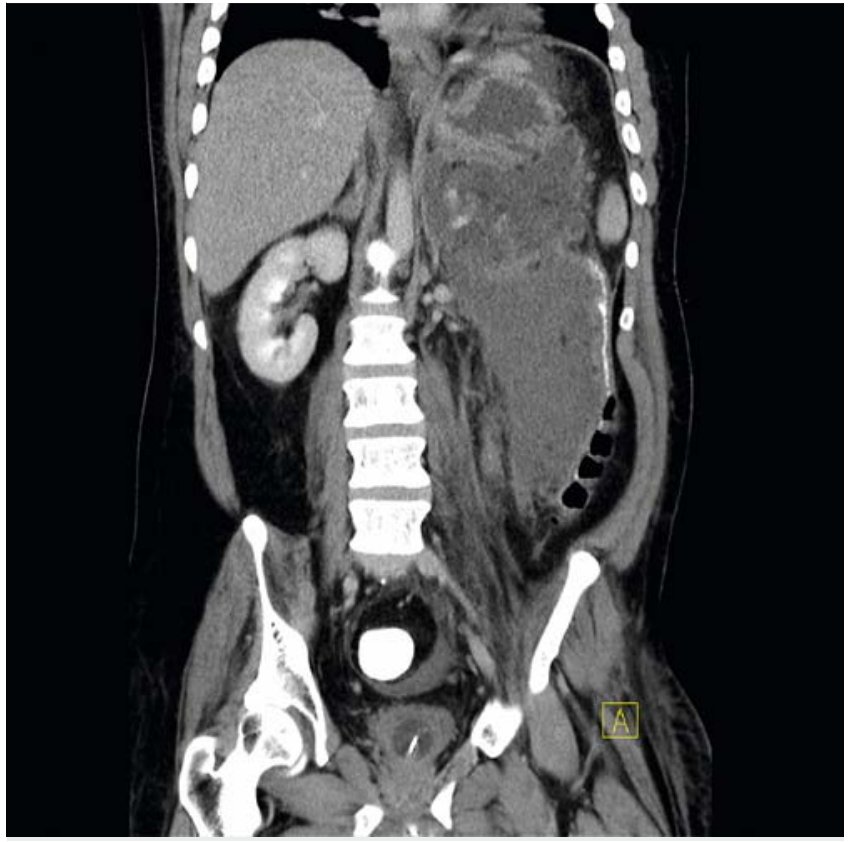

Fig. 5 Extensive pancreatic necrosis reaching the pelvis before therapy.

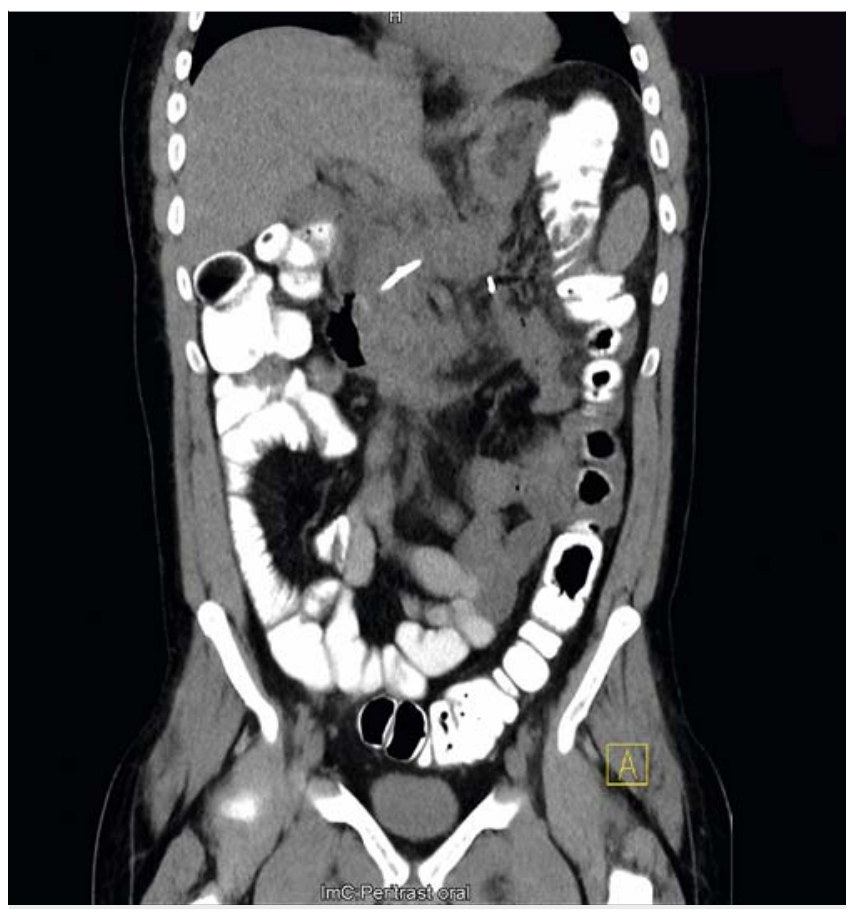

Fig. 6 Pancreatic necrosis 8 weeks after therapy.

The median CRP-level as a marker for systemic inflammation was $241 \mathrm{mg} / \mathrm{L}(182.8-288.9)$ and $23.3 \mathrm{mg} / \mathrm{L}(18-60)$ before and after therapy $(P<0.001)(\vee$ Table 2$)$.

The median time period for the 21 (43\%) patients who received intensive care was 5 days (3-9) in the ICU, time spent in the IMC was a median 3 days (2-4).
- Table 2 Intervention characteristics, clinical outcomes, and adverse events.

\begin{tabular}{|l|l|}
\hline & $\mathbf{n}=\mathbf{4 9}$ \\
\hline Technical success rate & $100 \%$ \\
\hline Clinical success rate & $87.8 \%$ \\
\hline Mortality & $8.2 \%$ \\
\hline Period until intervention $(\mathrm{d})$ median $(95 \% \mathrm{Cl})$ & $7(3-11)$ \\
\hline necrosectomies & $4(3-5)$ \\
\hline ICU treatment, $\mathrm{n}(\%)$ & $21(43 \%)$ \\
\hline ICU days, median $(95 \% \mathrm{Cl})$ & $5(3-9)$ \\
\hline IMC treatment, $\mathrm{n}(\%)$ & $33(67 \%)$ \\
\hline IMC days, median $(95 \% \mathrm{Cl})$ & $3(2-4)$ \\
\hline Follow-up(months), median $(95 \% \mathrm{Cl})$ & $14(10-18)$ \\
\hline CRP pre $(\mathrm{mg} / \mathrm{L})$, median $(95 \% \mathrm{Cl})$ & $241(182.8-288.9)$ \\
\hline CRP post $(\mathrm{mg} / \mathrm{L})$, median $(95 \% \mathrm{Cl})$ & $23.3(18-60)$ \\
\hline WBC pre $(\mathrm{T} / \mu \mathrm{L})$, median $(95 \% \mathrm{Cl})$ & $15.1(13.6-17.5)$ \\
\hline WBC post $(\mathrm{T} / \mu \mathrm{L})$, median $(95 \% \mathrm{Cl})$ & $7.9(6.5-9.3)$ \\
\hline Adverse events & $6(12.2 \%)$ \\
\hline Bleeding & $2(4.1 \%)$ \\
\hline Perforation & $2(4.1 \%)$ \\
\hline Dislocation & $4(8.2 \%)$ \\
\hline
\end{tabular}

ICU, intensive care unit; IMC, intermediate care unit; CRP, C-reactive protein; WBC, white blood cell.

\section{Adverse events}

One perforation combined with significant bleeding occurred and was handled endoscopically using an over-the-scope-clip (OTSC, Ovesco, Tuebingen, Germany). Two further clinically significant hemorrhages took place. One was managed endoscopically, the second patient was transferred to surgery because of recurrent hemorrhage in the necrotic cavity and died in the course of the disease due to multiple organ failure. Three stent dislocations occurred during endoscopic debridement. Those were successfully repositioned ( $\bullet$ Table 2 ). The median follow-up time was 14 months (10-18).

\section{Discussion}

In the course of acute necrotizing pancreatitis, the Atlanta classification distinguishes between the acute necrotic collection containing a various amount of fluid and necrotic tissue which is a criterion of an early stage of the disease and the walled-off necrosis consisting of necrotic material within an enhancing wall of reactive tissue which usually occurs in a later course of the disease [1] This encapsulated collection has a defined wall and matures over a time period $\geq 4$ weeks after onset of pancreatitis. Infected pancreatic necrosis is predominantly an attribute of this late phase of necrotizing pancreatitis. But in $25 \%$ of 
cases, infection of necrosis occurs within the first 14 days before the walled-off necrosis has fully matured [9].

In the case of early infected pancreatic necrosis, current guidelines recommend postponing the first intervention for 4 weeks after the onset of pancreatitis, if it is tolerated by the patient $[12,13]$. After demarcation of the infected tissue, drainage or debridement are supposed to be easier performed with fewer complications.

Different surgical based studies had shown an improved outcome by delaying the intervention for $\geq 4$ weeks [5-8]. Particularly stable patients might benefit from conservative treatment with antibiotics to delay the required intervention and give the necrotic collection time to evolve and demarcate.

In contrast, in the case of unstable, rapidly deteriorating patients, earlier intervention is inevitable. In this early stage of the disease, data about timing of the first intervention are sparse. A survey of 116 international pancreatologists showed a disagreement among the participants regarding the timing of invasive interventions: in the case of early infected necrosis, 55\% of respondents would postpone the intervention under antibiotic therapy, whereas $45 \%$ prefer immediate intervention [11].

The current European Society for Gastrointestinal Endoscopy guideline deliberates the early intervention in case of septic aggravation of the patient if a delay is not tolerable due to the patient's condition [10]. In our study, the patients received the first transluminal therapy after a median of 7 days (3-9, $95 \% \mathrm{Cl}$ ) after the first proof of necrosis.

At this particular time, most of the necrotic collections are not expected to be encapsulated. With regard to the previously published data, one would anticipate an increase in complication rate and eventually, mortality. In our study, despite postponing the intervention, the mortality rate was $8.2 \%$, which is comparable to previous studies ranging from $7.5 \%$ to $19 \%$ [ 8 , $14,15]$. These results suggest that early intervention is tenable when it is essential due to the clinical deterioration of the patient.

This is in contrast to earlier surgically based studies showing that postponing the interventions decreases mortality $[6,7$, 16]. Surgical necrosectomy of infected and not demarcated pancreatic necrosis resulted in distinct inflammatory stress leading to organ failure and increased mortality. But our data indicate that these surgical results are not entirely applicable to an endoscopically based approach.

Our results support the assumption that endoscopic intervention produces a minor inflammatory stimulus in comparison to surgery, which leads to a weakened systemic inflammatory response and enables intervention in the early phase of the disease, particularly when clinical deterioration due to infected pancreatic necrosis necessitates early intervention. In principle, this is in accordance with the results by Bakker et al., who demonstrated a reduction in the systemic inflammatory response in patients treated with endoscopic intervention [17].

Bang et al. compared an endoscopic transluminal approach with minimally invasive surgery and demonstrated a significantly lower rate of SIRS (20.6\% vs. $65.6 \%)$, and significantly fewer patients with new-onset SIRS (5.6\% vs. 56.3\%) 72 hours after endoscopic intervention [18]. This study included a high portion of patients in American Society of Anesthesiologists Class 3 and 4, and one- quarter of patients received their first endoscopic intervention within 4 weeks after the onset of pancreatitis. The low mortality rate of $8 \%$ in this study is in line with our study, which showed that an early timed endoscopic intervention is not only justifiable but often mandatory in patients with sepsis.

Our findings are also in line with the study by Trikudanathan et al., who compared an early ( $<4$ weeks) to a standard $(\geq 4$ weeks after onset of disease) endoscopically focused approach for necrotizing pancreatitis [19]. They found no difference in complication rates between the two patient groups and a slight increase in mortality for the early intervention (13\% vs. $4 \%$ ). The authors concluded that the early timed intervention is justified when the indication for it is strong.

Yan et al. compared immediate direct endoscopic necrosectomy after LAMS placement with necrosectomy after 1 to 2 weeks to allow the cystenterostomy to mature to reduce complications. They found no significant difference in procedure-related AEs between immediate and delayed intervention (7.5\% vs. 9.4\%) and demonstrated earlier resolution of pancreatic necrosis in the case of immediate necrosectomy [20].

This is consistent with our data showing comparable complication rates ( $>$ Table 2 ).

Four stent dislocations (8.2\%) occurred in this study. All incidents happened during the performance of necrosectomy. The stents were able to be retracted in the correct position using endoscopic forceps. LAMS offer the ability to enter the necrotic cavity easily without prior dilation. Stent dislocation during necrosectomy can be expected and does not imply an adverse outcome for the patient because it is recognized immediately.

The perforation rate $(4.1 \%)$ is in line with a systematic review, which included 455 patients in 14 studies (4\%) [21]. The bleeding risk was $5 \%$ in our study, in contrast to $18 \%$ in that review. This represents a complication rate associated with the early endoscopically based intervention comparable to a traditionally timed intervention.

Our results are limited by the low number of patients and the retrospective data analysis. It may be considered a strength that all patients with infected pancreatic necrosis and early endoscopic therapy admitted were included during the mentioned period. The study represents the entire patient population rather than a case series with selected patients suitable for endoscopic intervention.

\section{Conclusion}

In conclusion, our data show that in the case of infected pancreatic necrosis, endoscopic therapy can be performed earlier than 4 weeks if a patient's clinical status necessitates intervention. Further studies should be performed to delineate the optimal timing of endoscopic intervention.

\section{Competing interests}

The authors declare that they have no conflict of interest. 


\section{References}

[1] Banks PA, Bollen TL, Dervenis C et al. Classification of acute pancreatitis-2012: revision of the Atlanta classification and definitions by international consensus. Gut 2013; 62: 102-111

[2] Wu BU, Johannes RS, Sun X et al. The early prediction of mortality in acute pancreatitis: A large population based study. Gut 2008; 57: 1698-1703

[3] Beger HG, Rau BM. Severe acute pancreatitis: Clinical course and management. World J Gastroenterol 2007; 13: 5043-5051

[4] Petrov MS, Shanbhag S, Chakraborty M et al. Organ failure and infection of pancreatic necrosis as determinants of mortality in patients with acute pancreatitis. Gastroenterology 2010; 139: 813-820

[5] De Beaux AC, Palmer KR, Carter DC. Factors influencing morbidity and mortality in acute pancreatitis; an analysis of 279 cases. Gut 1995; 37: $121-126$

[6] Besselink MG, Verwer TJ, Schoenmakers EJ et al. Timing of surgical intervention in necrotizing pancreatitis. Arch Surg 2007; 142: 11941201

[7] Rodriguez JR, Razo AO, Targarona J et al. Debridement and closed packing for sterile or infected necrotizing pancreatitis: insights into indications and outcomes in 167 patients. Ann Surg 2008; 247: 294299

[8] Van Santvoort HC, Besselink MG, Bakker OJ et al. A step-up approach or open necrosectomy for necrotizing pancreatitis. N Eng J Med 2010; 362: $1491-1502$

[9] Petrov MS, Chong V, Windsor JA. Infected pancreatic necrosis: not necessarily a late event in acute pancreatitis. World J Gastroenterol 2011; 17: 3173-3176

[10] Arvanitakis M, Dumonceau JM, Albert J et al. Endoscopic management of acute necrotizing pancreatitis: European Society of Gastrointestinal Endoscopy (ESGE) evidence-based multidisciplinary guidelines. Endoscopy 2018; 50: 524-546

[11] Van Grinsven J, van Brunschot S, Bakker O] et al. Diagnostic strategy and timing of intervention in infected necrotizing pancreatitis: an in- ternational expert survey and case vignette study. HPB 2016; 18: 4956

[12] Besselink M, van Santvoort H, Freeman M et al. IAP/APA evidencebased guidelines for the management of acute pancreatitis. Pancreatology 2013; 13: e1-15

[13] Tenner S, Baillie J, deWitt ] et al. American college of Gastroenterology guideline: management of acute pancreatitis. Am J Gastroenterol 2013; 108: 1400-1415

[14] Seifert H, Biermer M, Schmitt W et al. Transluminal endoscopic necrosectomy after acute pancreatitis: a multicentre study with longterm follow-up (the GEPARD study). Gut 2009; 58: 1260-1266

[15] Van Brunschot S, van Grinsven J, van Santvoort H et al. Endoscopic or surgical step-up approach for infected necrotising pancreatitis: a multicentre randomised trial. Lancet 2018; 391: 51-58

[16] Hartwig W, Maksan SM, Foitzik T et al. Reduction in mortality with delayed surgical therapy of severe pancreatitis. J Gastrointest Surg 2002; 6: 481-487

[17] Bakker O], Santvoort HC, van Brunschot S et al. Endoscopic transgastric vs surgical necrosectomy for infected necrotizing pancreatitis: a randomized trial. JAMA 2012; 307: 1053-1061

[18] Bang JY, Arnoletti JP, Holt BA et al. An endoscopic transluminal approach, compared with minimal invasive surgery, reduces complication $s$ and costs for patients with necrotizing pancreatitis. Gastroenterology 2019; 156: 1027-1040

[19] Trikudanathan G, Tawfik P, Amateau S et al. Early ( $<4$ weeks) versus standard (>4 weeks) endoscopically centered step-up interventions for necrotizing pancreatitis. Am J Gastroenterol 2018; 113: 15501558

[20] Yan L, Dargan A, Nieto J et al. Direct endoscopic necrosectomy at the time of transmural stent placement results in earlier resolution of complex walled-off pancreatic necroisis: results from a large multicenter united states trial. Endosc Ultrasound 2019; 8: 172-179

[21] Van Brunshot S, Fockens P, Bakker OJ et al. Endoscopic transluminal necrosectomy in necrotizing pancreatitis: a systemic review. Surg Endosc 2014; 28: 1425-1438 